\title{
Un breve análisis en torno al uso de los tipos omisivos y la necesaria incorporación de la perspectiva de género en la aplicación de la dogmática penal
}

$A$ brief analysis of the use of omission types and the necessary incorporation of the gender perspective in the application of criminal dogmatics

Uma breve análise do uso dos tipos de omissão e a necessária incorporação da perspectiva de gênero na aplicação da dogmática criminal

Une brève analyse de l'utilisation des types d'omissions et de la nécessaire incorporation de la perspective de genre dans l'application de la dogmatique criminelle

遗漏的犯罪主体和性别

\author{
Yael Zygielman Tale \\ Universidad Nacional de La Plata - Argentina
}

Revista Derechos en Acción ISSN 2525-1678/ e-ISSN 2525-1686

Año 5/No 16 Invierno 2020 (21 junio a 20 septiembre), 277-290

DOl: https://doi.org/10.24215/25251678e424

Recibido: 01/03/2020

Aprobado: 01/04/2020

\footnotetext{
Abogada. Universidad Nacional de La Plata (UNLP) / Finalizando carrera de Especialización en Derecho Penal (UBA). Docente ayudante de primera categoría (interina) en la Cátedra I, de Derecho Penal I, a cargo del Dr. Slokar.W.A, de la Facultad de Ciencias Jurídicas y Sociales de la Universidad Nacional de la Plata. Directora de Lucha contra la Violencia Institucional de la Subsecretaria de Política Criminal del Ministerio de Justicia y Derechos Humanos. (ORCID: https://orcid.org/ 0000-0002-6810-803X).
} 
Resumen: El texto se propone complejizar desde una perspectiva crítica, el trato que los juzgadores dan a los delitos omisivos, cuando las que resultan imputadas son mujeres que se apartan de lo que consideran "un buen rol de madre". En estos supuestos bajo la apariencia del uso dogmático de la teoría, las sentencias recurren a argumentos derivados de la propia moral y en el análisis no tienen en consideración la historia de vida y la situación personal de estas mujeres.

Palabras clave: rol de madre, mujer, moral, perspectiva crítica.

Abstract: This work aims to make more complex, from a critical perspective, the treatment that judges give to omission crimes, when those who are accused are women who deviate from what they consider "a role as a good mother." In these cases, under the guise of dogmatic use of the theory, the sentences resort to arguments derived from morality itself and in the analysis they do not take into account the biography and particular situation of these women.

Keywords: maternal role, woman, moral, critical perspective.

Resumo: 0 texto visa tornar mais complexo, de uma perspectiva crítica, o tratamento que os juízes dão aos crimes de omissão, quando os acusados são mulheres que se desviam do que consideram "um bom papel de mãe". Nestes casos, a pretexto de uso dogmático da teoria, as frases recorrem a argumentos derivados da própria moralidade e na análise não levam em consideração a história de vida e a situação pessoal dessas mulheres.

Palavras-chave: papel da mãe, mulher, moral, perspectiva crítica.

Résumé: Le texte vise à rendre plus complexe, d'un point de vue critique, le traitement que les juges accordent aux crimes par omission, Iorsque les accusés sont des femmes qui s'écartent de ce qu'elles considèrent comme «un bon rôle de mère». Dans ces cas, sous couvert d'un usage dogmatique de la théorie, les phrases recourent à des arguments issus de la morale elle-même et dans l'analyse elles ne prennent pas en compte I'histoire de la vie et la situation personnelle de ces femmes.

Mot-clés: Rôle de la mère, de la femme, moral, perspective critique.

摘要: 案文旨在从批判性的角度出发, 使被指控的人是偏离了她们 认为的“母亲的好角色” 的妇女, 从而使法官对遗漏罪的处理更为复 
杂. 在这些情况下, 以教义主义为幌子, 这些句子诉诸于道德本身的 论点, 而在分析中, 它们没有考虑这些妇女的生活史和个人情况.

关键字: 母亲的角色, 女人, 道德, 批判的观点

\section{Un breve análisis en torno al uso de los tipos omisivos y la necesaria incorporación de la perspectiva de género en la aplicación de la dogmática penal}

En este trabajo complejizaremos desde una perspectiva crítica, el trato que los juzgadores dan a los delitos omisivos, cuando las que resultan imputadas son mujeres que se apartan de lo que consideran "un buen rol de madre". En estos supuestos bajo la apariencia del uso dogmático de la teoría, las sentencias recurren a argumentos derivados de la propia moral y en el análisis no tienen en consideración la historia de vida y la situación personal de estas mujeres.

Desde nuestra perspectiva la dogmática penal es una herramienta necesaria de construcción teórica que fue pensada para limitar la arbitrariedad del poder punitivo y, por ende, no debe ser usada para filtrar preconcepciones y estereotipos, por el contrario, debe tener en su horizonte de consideración el contexto en el que se desarrolla el hecho típico que se le atribuye a una persona y servir de contención a la arbitrariedad.

\section{Nuestra visión del Derecho y un posible horizonte}

Para comenzar este trabajo, es necesario puntualizar que partimos de la concepción de que el derecho es entendido no solo como conjunto de normas que regulan una sociedad determinada, sino como un artefacto de poder, que al igual que toda construcción de saber, no se define por ser una ciencia objetiva e imparcial, sino que por el contrario se inscribe y desarrolla en cierto contexto, de acuerdo a los valores de la época y a las estructuras de poder de cada sociedad. En este sentido si pensamos al saber jurídico como uno de los mecanismos de 
control de nuestras sociedades, no podemos pensarlo objetivo, sino que justamente en su pretensión de objetividad esconde su contenido performativo y regulador mediante el cual se sostienen estructuras de poder que fijan, invisibilizan y jerarquizan los géneros, regulando la forma de ser y participar en una sociedad determinada. Decimos que el saber jurídico y en este caso el derecho penal, invisibiliza porque al estructurarse binomicamente (hombre-mujer) niega otras identidades, fija porque establece el rol que compete a cada genero dentro del binarismo y jerarquiza, porque regula y sostiene relaciones de poder que resultan a per se, desiguales.

La perspectiva de género nos ayuda a complejizar sobre la falsa neutralidad de estos conceptos ideales, "buena esposa", "buena victima", "buena madre" ${ }^{2}$ conceptos naturalizados y utilizados por los juzgadores, que esconden bajo la pretensión de lo natural, una desigualdad. En este sentido los casos que analizaremos en este trabajo, traen consigo una carga de estereotipos, que buscan aleccionar a quienes se corren del "ideal de mujer" y de lo que se considera ser una "buena madre", creemos que no es la dogmática penal en este caso el problema en sí mismo, sino el mal uso que se hace de ella por parte de los juzgadores.

En este sentido encontramos que las mujeres participan de la agencia punitiva al menos de dos maneras: Como víctimas y como posibles autoras de delitos, aquí podríamos preguntarnos para complejizar quienes son seleccionadas la agencia penal $i a$ quienes se criminaliza y en función de que se lo hace? ¿cómo interviene la matriz de genero a la hora de criminalizar a las

2 La autora Carol Smart (1994) en el texto "la teoría feminista y el discurso jurídico" Entiende que el derecho opera como una tecnología del género, como un proceso de producción de identidad de género, en lugar de pensar que asigna roles a identidades ya constituidas. En este sentido "la Mujer", es una identidad simbólica, a la cual se le asigna determinadas características en contraposición al hombre y en contraposición a otras mujeres que se aparten de ese ideal (mujeres delincuentes, prostitutas, malas madres, malas víctimas). SMART, Carol. "La teoría feminista y el discurso jurídico». En BIRGIN, Haydée (comp.) El Derecho en el género y el género en el Derecho. Buenos Aires: Editorial Biblos, 2000. 
mujeres? ¿porque delitos se criminaliza más a este colectivo? ¿se lo hace en base a estereotipos? ¿Por qué las imputaciones resultan menos precisas?

De esta manera se puede ir construyendo estrategias que permitan visualizar y visibilizar que el género también debería ser una categoría de análisis real y concreta para el derecho penal, porque en lo óntico lo es. Invisibilizarlo bajo la pretensión de objetividad del derecho, jerarquiza una desigualdad que le prexiste.

\section{Una aproximación previa y necesaria: Los tipos penales}

Encontramos en nuestro Código Penal, en su parte especial la enumeración de variados delitos: En base a su estructura típica, los podemos dividir en delitos dolosos y culposos y a su vez pueden estos pueden ser activos u omisivos.

Cabe destacar que según la teoría a la que adherimos ${ }^{3}$, para el desarrollo de los delitos omisivos, partimos de la tipicidad de los mismos, por entender estos autores, que no existe a nivel pre-típico otras formas de expresión humana que no sean conductas (hacer dirigido a una finalidad determinada). En este encuadre la omisión resulta de naturaleza normativa. La omisión, consiste en un hacer diferente al ordenado por el tipo.

Según esta doctrina, en los delitos dolosos se sanciona a quien guio su finalidad, concretándola en un resultado querido y buscado. En los delitos culposos en cambio, lo que se sanciona es la violación a un deber de cuidado, cuando el sujeto, en la puesta en marcha de un proceso causal, viola un deber de cuidado que le indicaba actuar de determinada manera y produce un resultado lesivo.

En los delitos activos, el tipo penal describe art 79 C.P "el que matare a otro" un hacer, mientras que el mandato de la norma derivada del tipo enuncia un mandato de no hacer "no

\footnotetext{
3 Adherimos a la tesis funcionalista conflictiva de Zaffaroni, Alagia y Slokar.
} 
mataras". En los delitos omisivos en cambio el tipo penal describe de forma negativa la conducta 108 C.P "el que omitiere dar el auxilio necesario" y la norma deducida del tipo resulta imperativa, ordenando un hacer "auxiliaras". Cabe destacar que esta forma de entender la construcción de los tipos penales y la subsecuente teoría de las normas utilizada, es para una parte de la dogmática ${ }^{4}$ y no compartida por toda.

La estructura de los delitos de omisión dentro de la teoría del delito, es la estructura más endeble en su construcción teórica y menos investigada, la estructura mas desarrollada es la dolosa activa. Esto puede tener diversas causas, Zaffaroni plantea que su desarrollo dogmático comienza por los delitos dolosos activos porque son los mayormente tipificados en el código penal y en base a esa estructura desarrolla los siguientes, dejando poco lugar para los omisivos. Ello podría dar cuenta de la poca investigación que nuestra dogmática le ha dado a este tipo de delitos y la falta de armonización con el resto de las estructuras típicas, por ende, nos encontramos frente a un problema complejo.

A continuación, pondremos en tensión el uso que da la jurisprudencia a los delitos omisivos, para criminalizar a las mujeres que se apartaron de su rol de "buena madres", uso que creemos que justamente ocurre en virtud de la abstracción y falta de claridad y desarrollo dogmático en este tipo de estructuras.

\section{El dato óntico: Los casos de mujeres imputadas o sentenciadas bajo la modalidad omisiva, por violar su deber de garante: "Las malas madres"}

Cabe destacar, que el efecto derivado de la ausencia de la falta de perspectiva de género, lo pagan las mujeres criminalizadas, mediante encarcelamientos preventivos y sentencias fundadas en preconcepciones y estereotipos. Lo que no solo resulta

4 Manual de Derecho Penal Parte General Ed. Ediar,. 2006 Zaffaroni, Alagia y slokar 
injusto, sino que violenta las convenciones internacionales con jerarquía constitucional. ${ }^{5}$

El caso de Kalyna ${ }^{6}$, es una de las tantas sentencias en donde se juzga a las mujeres por el desempeño del rol de madre, pero también queremos mencionar el caso de Yanina Farias $^{7}$ acusada del homicidio agravado por el vínculo, cuando su pareja mata a su niño, pese a ser ella misma víctima de violencia, le imputan el delito de homicidio en la modalidad de comisión por omisión. Se la acusa por una pena más grave que al propio homicida al no intervenir en evitar el acontecimiento. Estuvo dos años en prisión preventiva, resulto absuelta. El caso de Yanina Gonzales ${ }^{8}$ es similar, acusada del delito de abandono de persona seguida de muerte y privada de la libertad preventivamente durante dos años.

Cecilia Hopp", expresa lo siguiente: "Estas imputaciones se basan en estereotipos e idealizaciones sobre los deberes de una buena madre, y omiten considerar la situación de la mujer. Se ignoran contextos de violencia habitual y los efectos de la violencia en la capacidad de las víctimas de cumplir con el rol esperado. La violencia contra las mujeres permanece invisible frente a la victimización de sus hijos y se sitúa a la madre como

5 Recordemos aquí que el derecho internacional marca la necesidad de que el accionar del Estado se encuentre libre de estereotipos. Tanto la "Convención Belém Do Para", como la CEDAW, obligan al Estado a la modificación de los patrones culturales que sustentan los estereotipos. En específico la recomendación $N^{0} 33$ de la CEDAW, encomienda a los Estados a que apliquen mecanismos que garanticen que las normas probatorias, investigaciones $y$ otros procedimientos probatorios jurídicos y cuasi judiciales sean imparciales y no estén influenciados por prejuicios o estereotipos de género.

6 Causa N 9125 -Sala II- "Kalyna, Silvia Noemí y otro s/ recurso de casación"

7 Para nombrar solo algunas, encontramos el reciente caso de Yanina Farias quien paso 2 años presa preventivamente acusada por omisión, en el delito de homicidio agravado por el vínculo, al no haber hecho nada para impedir que su pareja matara al niño: https://tn.com. ar/policiales/absolvieron-yanina-farias-la-mujer-que-estuvo-detenida-casi-dos-anos-por-noevitar-que-su-pareja_964289.https://www.pagina12.com.ar/185209-acusada

8 Causa 14.997 T.0.C 2 de San isidro.

9 Maternidad y delito: Una agenda para el feminismo. En Revista Argentina de Teoría Jurídica, Volumen 17 (marzo de 2017). 
victimaria. De esta forma, el derecho penal exige a las mujeres sacrificios heroicos para proteger a sus hijos e ignora los riesgos que suponen la separación y la huida. En definitiva, se minimizan los esfuerzos infructuosos de las mujeres para hacer cesar los ataques."

Kalyna $^{10}$ resulta condenada por el Tribunal Oral Criminal por abandono de persona, seguida de muerte agravado por el vínculo en el año 2007 y luego absuelta por el Tribunal de Casación Penal en el año 2013 (esta última revisión sí tuvo en consideración los antecedentes de violencia de genero sufridos a manos de su pareja y evaluó su historia de vulnerabilidad, donde se implicaban abusos sexuales, violencia y abandono ${ }^{11}$ ). Kalyna era madre soltera ${ }^{12}$ (vale mencionar que nunca se preguntaron los juzgadores dónde estaba el padre biológico del menor) ya que podríamos hablar allí de un abandono de persona por parte del padre, si usamos los mismos parámetros. Sin embargo, nadie se lo preguntaría.

Kalyna tenía a su cargo a un niño de 3 años y era trabajadora de un supermercado, el día del hecho concurre a su puesto de trabajo, dejando a su hijo al cuidado de quien fuera su pareja. Ella era el único sostén de familia y todo incumplimiento laboral repercutía en la percepción de su salario. Tenía planeado llevar a su hijo al médico luego de su jornada laboral. Ella no suponía que su niño se encontraba en un estado de peligro extremo (peritonitis aguda) a raíz de que el médico de la salita había visto al niño el día anterior, y no le había diagnosticado la peritonitis,

10 Causa N ${ }^{\circ} 9125$-Sala II- "Kalyna, Silvia Noemí y otro s/ recurso de casación"
11 El primer informe profesional respecto de Kalyna refiere a su historia de vida. Allí se informa
que sufrió malos tratos en el ámbito familiar durante su infancia, que ello motivó que quedara
al cuidado de sus abuelos, que durante aquel periodo su abuelo la violó, contando ella con
aproximadamente apenas cinco años de edad. Luego fue entregada junto con sus hermanos a
una familia sustituta, donde fue violada nuevamente y finalmente a sus ocho años, fue a vivir
con su padre (fs. $29 / 30$ del expte. 297/2005 del Juzgado Nacional en lo Civil N 102).

12 Según surge del fallo del Tribunal de Casación, en el voto del Dr. Slokar, en referencia a Kalyna quien "recordó que convivió con el padre biológico de M.K. durante tres años, pero que él la abandonó durante su embarazo". 
sino una afección hepática ${ }^{13}$ por ello Kalyna creía que el malestar del niño se debía solamente a un malestar hepático.

No obstante, el tribunal condena a Kalyna por abandono de persona seguida de muerte agravado por él vinculo. Considero que ella debía faltar a su trabajo, desconfiar de la palabra del médico de la salita y llevar nuevamente el niño a un especialista.

Según Hopp en "buena madre", "buena esposa", "buena mujer" donde aborda este caso: "El tribunal fundo su reproche sobre la base de la tranquilidad que evidenciaba la madre, a pesar de que su hijo se encontraba enfermo, asimismo, por haber antepuesto las obligaciones laborales antes de llevar al niño al médico. Los jueces de Casación observaron que el juicio se dirigió exclusivamente a evaluar la preocupación de KSN por su hijo y si se había mostrado consternada por su muerte, soslayando el interés por investigar los aspectos penales relevantes: si tenía dolo y quien había golpeado a la víctima" ${ }^{14}$.

¿No se le está pidiendo a estas mujeres un comportamiento heroico, en las circunstancias en las que se encontraban? ¿No se les está exigiendo una previsibilidad infundada e imposible en el ámbito de circunstancias reales en las que se encuentran? ¿El rol de madre se vuelve un criterio objetivo e ideal de actuación, que sobrepasa toda realidad óntica? ¿Se remplaza el conocimiento y la voluntad que exige el dolo, por un criterio objetivo fundando en el deber por ser madre? ¿Deber que

13 Según surge del fallo del Tribunal de Casación, en el voto del Dr. Slokar lo que sí se pudo acreditar es que Kalyna llevó al niño al médico y que el profesional aventuró que se trataría de una afección hepática, recomendó una consulta con un pediatra, pero no consideró que la urgencia fuera tal que mereciera el auxilio de una ambulancia. También resulta claro, a partir de los dichos médicos, que la sintomatología de la peritonitis puede ser confundida con otros problemas de salud menos peligrosos -tales como el malestar hepático o una indigestión-, dolencias que no requieren atención médica de urgencia y que, según una razonable representación, podían ser atendidas luego del horario de trabajo de Kalyna. Es por tal motivo que se presenta infundado el reproche referido a que la mujer hubiera concurrido a su trabajo en lugar de llevar a su hijo de inmediato al hospital.

14 Hopp, Cecilia "Buena Madre", "Buena Esposa", "Buena Mujer": abstracciones y estereotipos en la imputación penal. 
desconoce la historia y vivencia de cada mujer concreta y real y su ámbito de autodeterminación, el cual parte de situaciones constelacionales diferentes?

Son todas preguntas, que recorren la dogmática penal en sus diferentes estratos analíticos y que permite establecer un derecho penal de acto y humanista, que intenta dejar atrás un determinismo biológico y positivista, donde se juzga mecánicamente y de acuerdo a la peligrosidad que me brinda el derecho penal de autor. Por ello creemos, deben ser estas mismas preguntas, respetuosas de un derecho penal de acto, las que incorporen la perspectiva de género, para interrogar sobre las mujeres y sus circunstancias, y no juzgarlas de acuerdo a estereotipos, que no es más que un derecho penal de autor.

\section{El Remplazo del Dolo, por criterio objetivo de Buena Madre}

En la mayoría de estas sentencias se juzga bajo un criterio objetivo. La imputación se funda en el rol de buena madre, el cual resulta ser un criterio ideal, al que se acude, presumiendo y objetivando el dolo. Se antepone "el deber de madre" al conocimiento real y efectivo de la situación de peligro que requiere el tipo penal, por ejemplo, en la figura del abandono, artículo 106 C.P en su segunda modalidad "abandonar a su suerte a una persona" ${ }^{15}$ requiere que la persona conozca que está dejando a su suerte y en peligro a una persona, exigencias propias del dolo (conocimiento y voluntad) de lo que se realiza u omite o según otras teorías requerirá el conocimiento de la situación típica y la conciencia de la propia capacidad de acción ${ }^{16}$.

\footnotetext{
15 En las del art. 106 el núcleo es poner en peligro, colocando en situación de desamparo 0 abandonando a su suerte a una persona incapaz de valerse. En las del artículo 108 el núcleo está es omitir auxiliar.

16 "Si el dolo es aplicable a la omisión, puede afirmarse entonces que la voluntad de realización es aplicable a la omisión, por cuanto el conocer y querer las circunstancias del hecho son elementos fundamentales en casi todas las teorias del dolo." Cilleruelo, Alejandro R. “Delito de omisión” Edición 1. Fecha 2015. Pág. 92 in-fine y 94.
} 
Cuando se acude al criterio objetivo de buena o mala madre, se desconoce el hecho factico y las circunstancias que lo rodean, porque el rol de madre presupone que debía saber, conocer y proteger siempre y en todo momento a su hijo, aun cuando fácticamente no pudiera. Se implanta un dolo objetivo, derivado del rol y se le exige que debe conocer, aunque no lo haya hecho efectivamente. Se le exige más que a cualquier autor de delito, solo por el hecho de ser madre. A Kalyna por ejemplo, se le reprocho no haber llevado al niño a otro médico, haberse ido a trabajar, no volver a tiempo. ¿Y la finalidad de Kalyna dirigida a causar la muerte del niño, cuando se relevó?

Cabe recordar que en una imputación a titulo doloso, para la corriente a la que adherimos, la persona debe conocer su obrar y dirigirlo a una finalidad determinada.

Para una mayor claridad analizaremos los elementos requerido en los delitos omisivos:

Se requiere en un aspecto objetivo ${ }^{17}$ :

- Conocer la situación típica. (Que se abandona a alguien a quien se debe cuidar, 106 C.P)

- Exteriorización de una conducta diferente a la ordenada.

- La posibilidad física de realizar la conducta ordenada (posibilidad de evitar ese resultado, que se diferencia de la ausencia de conducta porque en este caso es la imposibilidad de realizar la acción ordenada, no toda acción) sin embargo en este punto hay controversias con otros autores.

- Nexo de evitación (un juicio hipotético que permite, establecer si actuando conforme la conducta ordenada, se hubiese evitado el resultado).

En un aspecto subjetivo:

- Se requiere el efectivo conocimiento de la situación típica (el abandono con su resultado, esto es la previsión

17 Según la teoría de Zaffaroni, Alagia y Slokar. 
del desencadenamiento del curso causal, que se está dejando morir, 106 C.P, por ejemplo, al realizar una conducta diferente a la ordenada, una decisión deliberada de no intervenir, la inminencia del peligro para el bien jurídico, la posibilidad fisca de intervención, la posición de garante)

- Se debe representar la realización de la conducta debida y la vía por la cual evitarlo.

- Otra parte de la doctrina plantea también la voluntad de: Perjudicar el bien jurídico, lo cual se lograr mediante el dominio del propio cuerpo (en su forma de inactividad) como medio para que se produzca un resultado. Con la finalidad requerida (voluntad final de permanecer inactivo).

Todos son elementos necesarios para construir una imputación penal por omisión, pero en estas sentencias observamos que nada de ello es tenido en cuenta. Por el contrario, el dato de realidad, nos arroja que se elaboran sentencias irracionales, que desconocen las circunstancias concretas de las personas concretas, y se presumen conductas dolosas, fundadas en roles abstractos.

Es paradigmático que en un principio la posición de garante de la que derivan el rol, fue usada para limitar la expansión del derecho penal, al limitar el circulo de autores a ciertos casos, y hoy en día esa posición es usada para ampliar los márgenes limitantes del dolo. ${ }^{18}$.

\section{Algunas conclusiones}

A modo de síntesis, concluimos que el derecho no es objetivo, ni avalorado, y el análisis de algunos casos, en particular el de Kalyna, nos demostró que se condena a mujeres en base

18 En relación a las deficiencias del dolo, Cilleruelo expresa que "La cuestión intenta matizarse, sin éxito, construyendo un concepto artificial que se ha dado en llamar cuasi dolo, para dotar a la imputación de un aspecto subjetivo que en realidad así planteado, es solo aparente." Cilleruelo, Alejandro R. "Delito de omisión" Edición 1. Fecha 2015 Pag 135. 
a estereotipos. Que cuando se trata de delitos omisivos, esto se potencia aún más, al resultar los tipos omisivos, tipos abiertos que por su estructura (requieren de una valoración por parte de los jueces, para determinar el deber de garante en cada caso)

El deber de garante en estos casos, es fundado en preconceptos sobre los que es un buen rol de madre (lo que además suponen objetivo y avalorado per se) y se condena a mujeres, sin demostrar intención alguna (dolo) de cometer ilícito penal. Es la madre (quien todo lo puede y todo lo debe) quien no cumplió con su rol, quien no impidió el resultado, la responsable, sin importar si quiso o no quiso, exigiéndole conductas heroicas, a modo que enunciaba Hoff.

Los procedimientos judiciales que analizamos, ya sea con meras imputaciones o sentencias firmes, se realizan sin elementos jurídicos que vinculen el accionar/omitir con el resultado acaecido, no respetan elementos mínimos y necesarios para realizar una imputación penal, o confirmada la imputación, no tienen en consideración ámbitos de autodeterminación muy restringidos.

También suelen fundarse en "deberes morales" no admitidos por nuestra Carta Magna, los que resultan extensivos de punibilidad y violan el principio de legalidad formal.

Esto sucede tanto con los delitos tipificados en el código penal en la modalidad omisiva, que para una parte de la doctrina son los únicos constitucionales, pero también sucede cuando se realizan imputaciones en la modalidad de comisión por omisión.

Concluimos que producto de una moral determinada y fundada en estereotipos, estas sentencias hacen un uso erróneo de la construcción dogmática del deber de garante, para fundar sentencias injustas que nada tienen que ver con un derecho penal de acto, se olvidan del dolo como elemento reductor y necesario y proceden a una imputación por mera responsabilidad objetiva, contraria a cualquiera manifestación de un derecho penal elemental. 


\section{Bibliografía}

Arduino, Ileana (2018) "Los hilos punitivos de la trama neoliberal: intercambios en clavefeminista" en Emergencias: repensar el estado, las subjetividades y la acción política. Editorial Ciccus. Buenos Aires

Bodelon, Encarna (2003) Género y sistema Penal: los derechos de las mujeres en el sistema penal. En línea en: http://www. ucipfg.com/Repositorio/MCSH/MCSH10/Bloque_academico/ Unidad03/Genero_sistema_penal_derechos_mujeres_Bodelon.pdf

Cilleruelo, Alejandro (2015) Delito de omisión. Editorial Cathedra jurídica. Buenos Aires.

Gracia, Luis Martín (2004) El finalismo como método real sintético, para la construcción de la teoría de la responsabilidad penal. En línea en: www.criminet.ugr.es/recpc/06/recpc06-07.pdfm

Hopp, Cecilia (2017) "Buena madre, buena esposa y buena mujer", en Género y justicia penal. Editorial Didot. Buenos Aires.

Olsen, Frances (2000) "El sexo del derecho", en Identidad femenina y discurso jurídico. Editorial Blos. Buenos Aires.

Zaffaroni, Eugenio Raúl (1981) Manual de derecho penal: parte general. Editorial Ediar. Buenos Aires 